\title{
A Planar Generator for a Wave Energy Converter
}

\begin{tabular}{|r|l|}
\hline Journal: & Transactions on Magnetics - Conferences \\
\hline Manuscript ID & MAGCON-18-11-1678.R1 \\
\hline Manuscript Type: & MMM-Intermag 2019 \\
\hline Author: & 07-May-2019 \\
\hline & $\begin{array}{l}\text { Complete List of Authors: } \\
\text { Cranto, Domenitta, Vincenzo; Università degli studi di Palersità degli studi di Palermo, DEIM DIM } \\
\text { Liu, Zhenwei; RMIT University } \\
\text { Wang, Xuk; RMIT University }\end{array}$ \\
\hline Keywords: & \begin{tabular}{l} 
81 Electrical machines \\
\hline
\end{tabular} \\
\hline
\end{tabular}

\section{SCHOLARONE ${ }^{m}$ Manuscripts}




\title{
A Planar Generator for a Wave Energy Converter
}

\author{
Marco Trapanese ${ }^{1}$, Senior Member, IEEE, Domenico Curto ${ }^{1}$, Vincenzo Franzitta ${ }^{1}$, Member, IEEE, Zhenwei Liu ${ }^{2}$, \\ Luke McNabb ${ }^{2}$, Student Member, IEEE, Xu Wang ${ }^{2}$, \\ ${ }^{1}$ DEIM, Palermo University, Palermo, 90128, Italy \\ ${ }^{2}$ School of Engineering, Royal Melbourne Institute of Technology Melbourne VIC 3000, Australia
}

\begin{abstract}
This paper presents a permanent magnet planar translational generator which is able to exploit multiple modes of sea wave energy extraction. Linear electrical generators have recently been studied for the exploitation of sea wave energy, but, to the best of our knowledge, no synchronous planar translational generator has been proposed. In this paper, to maximise the energy extraction, we have considered all the potential modes of motion due to wave excitation and included them within the mathematical model of the proposed system. The principle of operation of the generator can be summarized as follows: the moving part (translator) of the generator is driven from the sea waves and induces and EMF on the windings mounted to the armature. The movement of the translator is two dimensional and therefore all the movement modes of the wave, except heave, can be exploited. The proposed mathematical model includes the dynamic equations of the translator and the electric equations of the windings. The coupling parameters (inductances, fluxes etc.) have been determined by FEM analysis. Optimization of the device has been performed by considering both, the parameters of the electromagnetic circuit, and, the parameters associated with the stochastic features of the wave.
\end{abstract}

\section{Index Terms-Electric machines, linear generators, Wave Energy Converters.}

\section{INTRODUCTION}

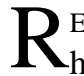
ECENTLY several approaches to harvest sea wave energy have been proposed; almost all of them introduce an intermediate mechanical conversion device between the waves and the electromagnetic generator. This stage converts the mechanical energy contained in the excited modes of motion of the converter (i.e. heave, sway, surge, yaw, pitch and roll) into a more usable form. This coupling device introduces additional losses, reduces system reliability, and adds additional maintenance requirements. In order to solve these issues, a generator directly coupled to the sea waves entirely contained in a vessel has been proposed. These systems are known as Inertial Wave Energy Converters, and until now, they have been designed to exploit only one mode of motion from the wave energy converter. A majority of the proposed solutions are based on point absorber technology coupled to a linear generator to extract energy via the heave motion, or, by a rotating generator coupled to a flywheel for rolling motions. This paper presents a permanent magnet planar translational generator which is able to exploit multiple modes of motion.

Recently, linear electrical generators have been studied with a reasonable intensity for the exploitation of sea wave energy [1-4]. A single initial study using a switched reluctance planar machine for wave energy harvesting was proposed in [5], but, to our best knowledge, no synchronous planar translational generators have been proposed. In this paper, in order to maximise the energy extraction, all the motion modes of the waves have been considered and included in the mathematical model of the system. The principle of operation of the generator can be summarised as follows: the moving part (translator) of the generator is inertially driven from the motion of the buoy and induces and EMF on the winding mounted on the armature. The movement of the translator is two dimensional and therefore all the movement modes of the wave, but heave motion can be exploited. The proposed mathematical model includes the dynamic equations of the moving part of the generator and the electric equations of the windings. The coupling parameters (inductances, fluxes etc.) have been determined by a FEM analysis. An initial approach to the optimization of the device has been performed by examining the parameters of the electromagnetic circuit. However, the optimization of the generator should be considered further during subsequent design analysis.

\section{CHARACTERISTICS OF SEA WAVES}

The classification of marine waves can be based on several approaches [4]. Most of which characterize the wave based on wavelength and period. In Table 1 , the different types of

TABLE 1: OCEAN WAVE CLASSIFICATION

\begin{tabular}{|c|c|c|}
\hline Classification & Period & Generating/Restoring Force \\
\hline Capillary waves & $<0.1 \mathrm{~s}$ & Wind/surface \\
\hline Ultragravity waves & $0.1-1 \mathrm{~s}$ & Wind/surface tension and gravity \\
\hline Gravity waves & $1-20 \mathrm{~s}$ & Wind/Gravity \\
\hline Infragravity waves & $20 \mathrm{~s}-5 \mathrm{~min}$ & $\begin{array}{l}\text { Wind and atmospheric pressure } \\
\text { gardients/Gravity }\end{array}$ \\
\hline Long-period waves & $5 \mathrm{~min} / 12 \mathrm{~h}$ & $\begin{array}{l}\text { Atmospheric pressure gradients and } \\
\text { earth quake/Gravity }\end{array}$ \\
\hline Ordinary tidal waves & $12-24 \mathrm{~h}$ & $\begin{array}{l}\text { Gravitational attraction/Gravity and } \\
\text { Coriolis Force }\end{array}$ \\
\hline Transtidal wave & $>24 h$ & $\begin{array}{l}\text { Storms and gravitational } \\
\text { attraction/Gravity and Coriolis } \\
\text { Force }\end{array}$ \\
\hline
\end{tabular}

surface waves are presented with respect to both the wave period and the associated originating forcing, as well as the restoring mechanisms.

All of these waves present with some form of stochastic features and are described in terms of mean wave amplitude, mean wave period and the direction of propagation. In real conditions, all parameters are constantly changing, and the waves are to be considered non-sinusoidal. As a result, a real floating body is subjected to several disturbing forces and 


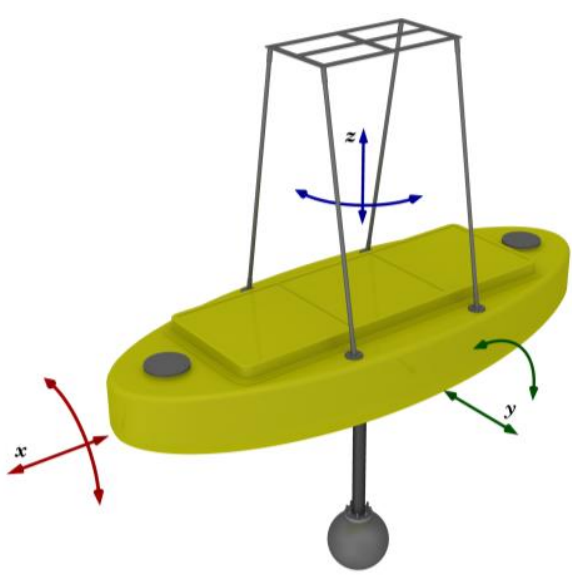

Figure 3: Motion modes of a floating body.

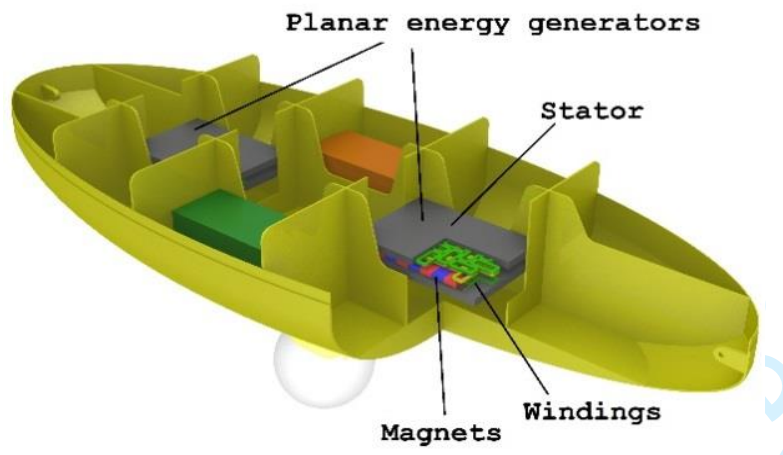

Figure 1: Planar generators inside a floating buoy.

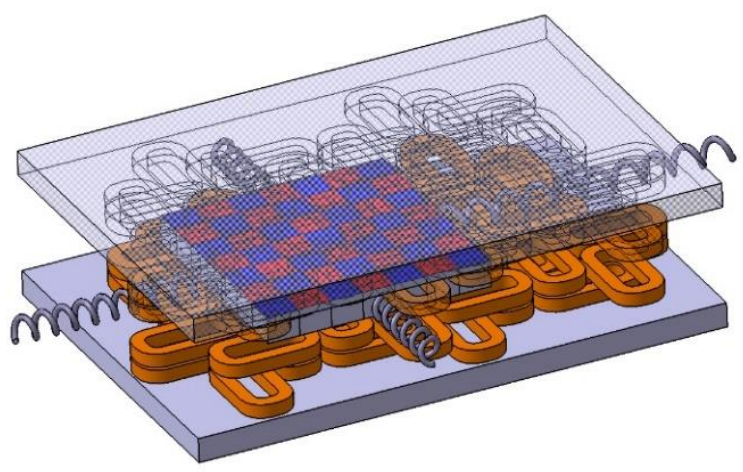

(a)

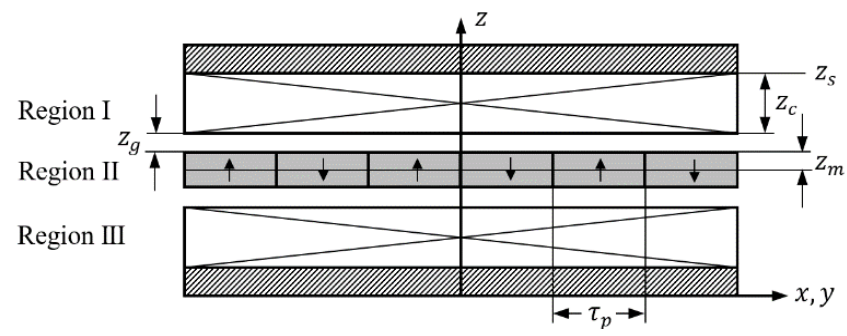

(b)

Figure 2: (a) 3D Model of the machine. (b) Planar machine cross-section geometry. The machine has three regions corresponding to air-gaps where the coil windings are distributed (regions I and III), and the area of the permanent magnet array (region II). torques that can excite several motion modes. Fig.1 illustrates all the possible motion modes of a floating body. To date, most of the proposed sea wave generators tend to exploit only one degree of freedom in order to produce electrical power. In this paper, we introduce a planar generator that can exploit two degrees of freedom of the movement from the floating body, as shown in Fig. 2. The principle of operation of the generator can be summarised as follows: the buoy is driven from the sea waves; as a result, the planar translator slides inside the armature, which is directly mounted on the frame of the buoy and is composed by the windings and induces an EMF on the windings. The movement of the translator is two dimensional, therefore at least rolling and pitching motion can be easily exploited. Several springs (Fig. 3) are also connected to the translator in order to avoid any strong impact with the surrounding frame.

\section{GEOMETRIC STRUCTURE OF THE PLANAR GENERATOR}

The general topology of the proposed planar machine consists of a symmetric dual layer stator made from a soft ferromagnetic material and a magnetic translator fitted between the stator. A three-dimensional diagram and cross section is shown in Fig.3a and b. The magnetic translator will be made from permanent magnet material, as is typically required by synchronous direct drive wave energy conversion systems due to size and weight constraints [6]. The coils are mounted to the stator material symmetrically on either side of the translator to ensure that the $z$-axis force components cancel. An ' $n$ ' phase machine can be developed by keeping each symmetric coil pair separate, or a standard three-phase structure can be formed by placing coils in series/parallel as desired. The former has potential benefits from an efficiency and controllability perspective as coil excitation only occurs when near the magnetic translator; however, the latter is more convenient from a standard power electronics and control aspect $[7,8]$.

The proposed machine structure also must take into account the practicalities of a complete wave energy system. Two specific areas that need to be considered when selecting the topology are: (a) the stochastic features (frequency, period and amplitude) of the wave energy source, as well as the standard range of input modes to the machine (e.g. roll, pitch, surge, etc.); (b) controllability of the machine to enable optimisation of the mechanical resonance in light of issue (a).

As a permanent magnet machine, no machine level control can be achieved (e.g. field winding control) in order to ensure that the machine can cater for the broad-band of wave input variation (see Table 1). Consequently, each symmetric coil pair on either side of the translator will need to be coupled to a bi-directional power electronic converter to moderate the machine output force and hence optimise the absorbed power in real time concerning the input wave. In conjunction with this active control, tuned springs can be attached to the translator to assist with maintaining mechanical resonance as shown in Fig.3a. This energy is then transferred to a DC bus by the converter where it is ready for cross-sea transport then grid connection via a final power electronic inversion stage. 
Alternatively, the DC supply can be directly converted into a different fuel source such as hydrogen [9].

In general, to address the discussed issues the machine will require force control regulated by a power electronic stage. However, due to the two-dimensional motion of the machine the force regulation scheme demands specific geometric requirements. With only one control variable per coil, being the electrical current, we require that each coil effectively produce force in only one dimension. One of the winding pitch dimensions must hence be more significant than the other to create a "decoupled" dominant force vector (i.e. $\tau_{w p x} \gg \tau_{w p y}$ or $\tau_{w p y} \gg \tau_{w p x}$; see Fig.4 for dimensions) [7]. To cater for this requirement, the following topological selections are made: (a) a dual-layer "herringbone" coil structure is used to ensure force control in both dimensions at all positions is achievable (see Fig.4); this structure is commonly seen structure in machines which require high precision force control in multiple dimensions [10]; (b) a slottless structure is selected due to the complex nature of the required winding pattern; this indicates that it will be preferable to keep the coil windings to a minimal $z$ axis pitch $\left(\tau_{w z}\right)$ to reduce the leakage inductance and maintain the flux density through the coils.

\section{ANALYTICAL SOLUTION}

\section{A. Magnetic Field Distribution}

The following section details the development of an analytical solution to the magnetic field distribution throughout the spatial regions of the planar generator as depicted in Fig. 3b. Within this solution the following assumptions are made:

1) The $x$ and $y$ dimensions of the machine are infinite so that the magnetic field is periodically distributed in these dimensions.

2) For the proposed topology, the stator dimensions are significantly larger than the magnetic translator. Hence the associated fringing effects are considered negligible.

3) The stator core is made of an ideal, soft ferromagnetic material with an infinite relative permeability.

4) The field is quasi-static and analyzed under an open circuit; i.e. the free current $\left(\mathbf{J}_{f}\right)$ is zero.

Fig. 3b illustrates that the field has three distinct regions: regions I and III are air-gaps where the coil windings are distributed, and region II is the permanent magnet space. For each of these regions, an appropriate boundary value problem can be developed to solve the magnetic field distribution. Note, region III is a reflection of region I in the $x-y$ axis with a phase shift by a pole pitch $\tau_{p}$ in the same planes. The solution to the field distribution in region III is not presented due to this symmetric property as it can be easily extrapolated from the solution of region I. For each region we desire solutions to the following:

$$
\mathbf{B}=\left\{\begin{array}{l}
\mu_{0} \mathbf{H}_{\mathrm{I}} \\
\mu_{0}\left(\mathbf{H}_{\mathrm{II}}+\mathbf{M}_{\mathrm{II}}\left(\mathbf{H}_{\mathrm{II}}\right)\right)=\mu_{0} \mu_{r \mathrm{II}} \mathbf{H}_{\mathrm{II}}+\mu_{0} \mathbf{M}_{0 \mathrm{II}}
\end{array}\right.
$$

where $\mu_{r I I}$ is the relative permeability of the permanent magnet and $\mathbf{M}_{\mathrm{II}}(\mathbf{H})$ is a general nonlinear magnetisation vector. For a "hard" magnet, the nonlinear hysteresis mechanism which sets the remanent "permanent-field" inside
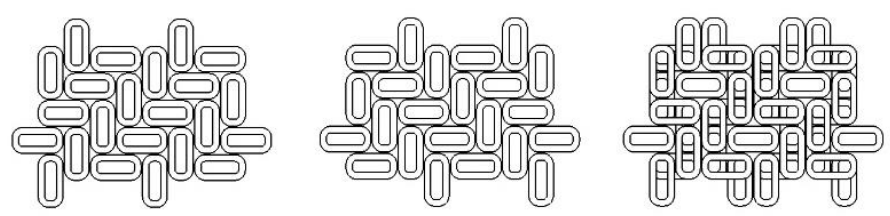

(a)

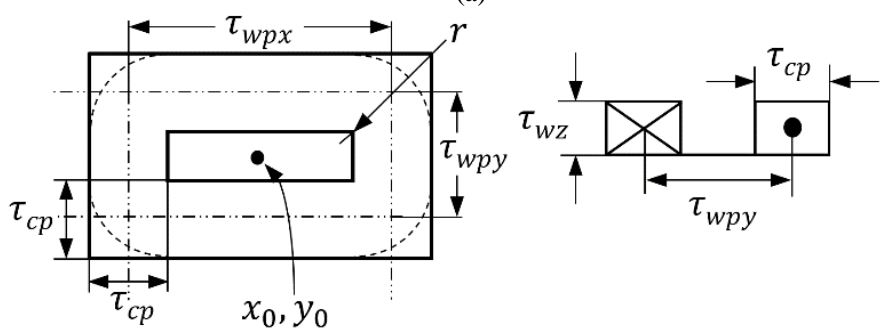

(b)

Figure 4: (a) Herringbone coil pattern; left: bottom layer; middle: top layer; right: combined. (b) Individual coil geometry. See Fig.3a for in region illustration.

the material can be approximated by a constant, $\mathbf{M}_{0 \mathrm{II}}$, plus the magnetization due to an applied field, $\mathbf{M}_{\mathrm{II}}$, assuming a perturbation of the magnetic field is sufficiently linear; i.e. $\mathbf{M}_{\text {II }}\left(\mathbf{H}_{\mathrm{II}}\right)=\mathbf{M}_{\mathrm{II}}+\mathbf{M}_{0 \mathrm{II}}$.

The permanent magnetisation vector $\mathbf{M}_{0 \mathrm{II}}$ is a twodimensional array of square magnets in an alternating NorthSouth pattern as depicted in Fig. 3a. The distribution, which only projects into the $z$-direction along the $x-y$ plane can be described as [11]:

$$
\mathbf{M}_{0 \mathrm{II}}=M_{0 \mathrm{II}} \hat{z}=\sum_{n=1}^{\infty} \sum_{k=1}^{\infty} \frac{B_{r e m}}{\mu_{0}} M_{z n} M_{z k} \cos \left(m_{n} x\right) \cos \left(m_{k} y\right) \hat{z}
$$

where $B_{r e m}$ is the permanent magnet remanence, $\hat{z}$ is a unit vector in the $z$ direction and:

$$
\begin{gathered}
M_{z n}=\frac{4}{\tau_{p} m_{n}} \sin \left(\frac{m_{n} \tau_{m}}{2}\right) \\
M_{z k}=\frac{4}{\tau_{p} m_{k}} \sin \left(\frac{m_{k} \tau_{m}}{2}\right) \\
m_{n}=\frac{(2 n-1) \pi}{\tau_{p}} \\
m_{k}=\frac{(2 k-1) \pi}{\tau_{p}}
\end{gathered}
$$

It is assumed that the magnet pitch $\tau_{m}$ covers the whole pole pitch $\tau_{p}$ for the topology used in this paper.

Since there is no free current in the system it is possible to evaluate the $\mathbf{H}$ field of each region in terms of a scalar potential; i.e.:

$$
\mathbf{H}=-\nabla \Phi_{\mathrm{M}}
$$

Given that the divergence of a magnetic field is zero, the solution for the scalar potential in region II is found from the following Poisson equation in three dimensions:

$$
\nabla^{2} \Phi_{\mathrm{M}}=\nabla \cdot \mathbf{M}_{0 \mathrm{II}}=0
$$

As a matter of convenience, the divergence of the permanent magnetisation vector is zero. Hence the Poisson equation simplifies down to a Laplace equation. 
By solving the Laplace equation in region $\mathrm{I}$, it is possible to directly compute the magnetic field $\mathbf{B}_{I}$; i.e.:

$$
\begin{gathered}
\mathbf{B}_{\mathrm{I}}=-\nabla \Psi_{\mathrm{M}} \\
\nabla^{2} \Psi_{\mathrm{M}}=0
\end{gathered}
$$

The following boundary conditions can be imposed to define unique solutions:

$$
\begin{gathered}
B_{x I}\left(x, y, z_{s}\right)=0 \\
B_{z I}\left(\tau_{p}, y, z\right)=B_{z I}\left(-\tau_{p}, y, z\right) \\
B_{z I}\left(x, \tau_{p}, z\right)=B_{z I}\left(x,-\tau_{p}, z\right) \\
H_{x I}\left(x, y, z_{m}\right)=H_{x I I}\left(x, y, z_{m}\right) \\
B_{z I}\left(x, y, z_{m}\right)=B_{z I I}\left(x, y, z_{m}\right) \\
H_{z I I}\left(\tau_{p}, y, z\right)=H_{z I I}\left(-\tau_{p}, y, z\right) \\
H_{z I I}\left(x, \tau_{p}, z\right)=H_{z I I}\left(x,-\tau_{p}, z\right)
\end{gathered}
$$

Solving (1) using the results from (4)-(5) with the specified boundaries in (7a-g), yields the following vector component solutions for the magnetic field distribution:

$$
\begin{aligned}
B_{x I} & =\sum_{n=1}^{\infty} \sum_{k=1}^{\infty} a_{\text {Ink }}^{\prime} m_{n} \sin \left(m_{n} x\right) \cos \left(m_{k} y\right) \sinh \left(m_{\epsilon}\left(z-z_{s}\right)\right) \\
B_{y I} & =\sum_{n=1}^{\infty} \sum_{k=1}^{\infty} a_{\text {Ink }}^{\prime} m_{k} \cos \left(m_{n} x\right) \sin \left(m_{k} y\right) \sinh \left(m_{\epsilon}\left(z-z_{s}\right)\right) \\
B_{z I} & =\sum_{n=1}^{\infty} \sum_{k=1}^{\infty}-a_{I n k}^{\prime} m_{\epsilon} \cos \left(m_{n} x\right) \cos \left(m_{k} y\right) \cosh \left(m_{\epsilon}\left(z-z_{s}\right)\right)
\end{aligned}
$$$$
B_{x I I}=\sum_{n=1}^{\infty} \sum_{k=1}^{\infty} a_{I I n k}^{\prime} m_{n} \sin \left(m_{n} x\right) \cos \left(m_{k} y\right) \sinh \left(m_{\epsilon} z\right)
$$$$
B_{y I I}=\sum_{n=1}^{\infty} \sum_{k=1}^{\infty} a_{I I n k}^{\prime} m_{k} \cos \left(m_{n} x\right) \sin \left(m_{k} y\right) \sinh \left(m_{\epsilon} z\right)
$$

$B_{Z I I}=\sum_{n=1}^{\infty} \sum_{k=1}^{\infty}-a_{I I n k}^{\prime} m_{\epsilon} \cos \left(m_{n} x\right) \cos \left(m_{k} y\right) \cosh \left(m_{\epsilon} z\right)$

$$
+B_{r e m} M_{z n} M_{z k} \cos \left(m_{n} x\right) \cos \left(m_{k} y\right)
$$

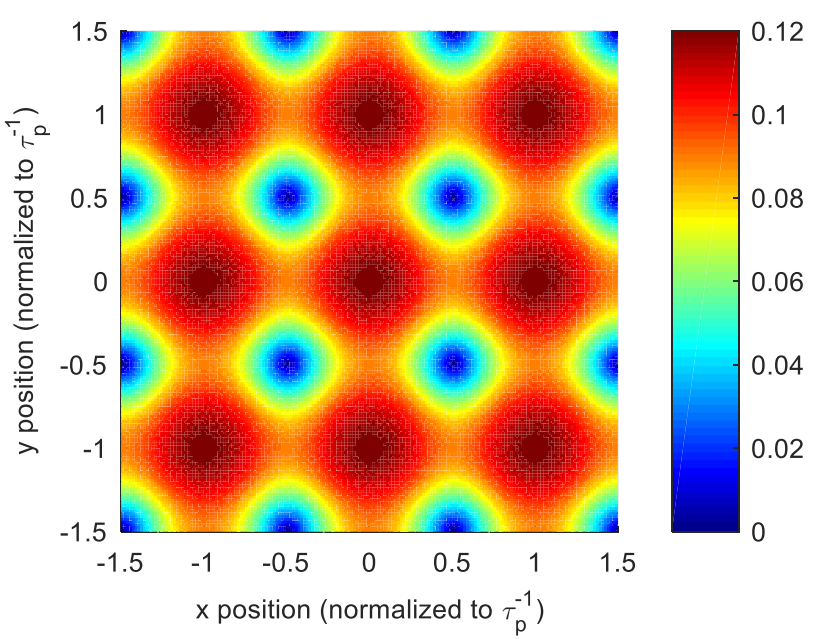

Figure 5: Analytical $|\mathbf{B}|$ distribution at $\mathrm{z}=\mathrm{z}_{\mathrm{m}}+\left(\mathrm{z}_{\mathrm{g}}+\mathrm{z}_{\mathrm{c}}\right) / 2$ of internal $3 \times 3$ magnets.
TABLE 2: GEOMETRIC RATIOS AND MAGNETIC PARAMETERS

\begin{tabular}{|l|c|c|c|c|c|}
\hline \hline Parameters & $z_{m} / \tau_{p}$ & $z_{s} / z_{m}$ & $\mu_{r I}$ & $\mu_{r I I}$ & $B_{r e m}$ \\
\hline Ratio & 0.3333 & 3.6 & 1.0 & 1.0998 & 1.2300 \\
\hline
\end{tabular}

Where:

$$
\begin{gathered}
a_{\text {Ink }}^{\prime} \\
=\frac{B_{r e m} M_{z n} M_{z k}}{m_{\epsilon} \cosh \left(m_{\epsilon}\left(z_{m}-z_{s}\right)\right)} \frac{\tanh \left(m_{\epsilon} z_{m}\right)}{\tanh \left(m_{\epsilon} \mathrm{z}_{\mathrm{m}}\right)-\tanh \left(m_{\epsilon}\left(\mathrm{z}_{\mathrm{m}}-\mathrm{z}_{\mathrm{s}}\right)\right)} \\
a_{\text {IInk }}^{\prime}=a_{\text {Ink }}^{\prime} \frac{\mu_{r I I} \sinh \left(m_{\epsilon}\left(z_{m}-z_{S}\right)\right)}{\mu_{r I} \sinh \left(m_{\epsilon} z_{m}\right)} \\
m_{\epsilon}=\sqrt{m_{n}^{2}+m_{k}^{2}}
\end{gathered}
$$

\section{B. Flux Linkage and EMF Prediction}

Utilizing the evaluated magnetic field distribution, an analytical prediction can be found to estimate the flux linkage and voltage generated per coil winding. For a coil with $N_{c}$ windings and finite dimensions (see Fig.4), the flux linkage can be estimated by evaluating the average value over the coil dimensions. There are effectively three surfaces contributing to the flux for the coil due to the finite pitches, i.e. $d x d y \hat{z}$, $d x d z \hat{y}$, and $d y d z \hat{x}$. The estimated flux over each surface can be found as follows:

$\Phi_{c x y}$

$=\frac{N_{c}}{\tau_{c p}^{2} \tau_{w z}} \int_{z_{m}+z_{g}}^{z_{m}+z_{g}} \int_{\tau_{x i}}^{+\tau_{w o}} \int_{\tau_{y i}}^{\tau_{y o}}\left(\int_{x-\frac{\tau_{x}}{2}}^{x+\frac{\tau_{x}}{2}} \int_{y-\frac{\tau_{x}}{2}}^{y+\frac{\tau_{x}}{2}} B_{z I} d y d x\right) d \tau_{y} d \tau_{x} d z$

$\Phi_{c x z}$

$=\frac{N_{c}}{\tau_{c p}^{2} \tau_{w z}} \int_{\tau_{x i}}^{\tau_{x o}} \int_{x-\frac{\tau_{x}}{2}}^{x+\frac{\tau_{x}}{2}} \int_{\tau_{y i}}^{\tau_{y o}}\left(\int_{z_{m}+z_{g}}^{z_{m}+z_{g}} \int_{y-\frac{\tau_{x}}{2}}^{y+\frac{\tau_{x}}{2}} B_{x I} d y d z\right) d \tau_{y} d x d \tau_{x}$

Where: $\tau_{x i}=\frac{\tau_{w p x}}{2}-\frac{\tau_{c p}}{2}, \quad \tau_{x o}=\frac{\tau_{w p x}}{2}+\frac{\tau_{c p}}{2}, \quad \tau_{y i}=\frac{\tau_{w p y}}{2}-\frac{\tau_{c p}}{2}$ and $\tau_{y o}=\frac{\tau_{w p y}}{2}+\frac{\tau_{c p}}{2}$.

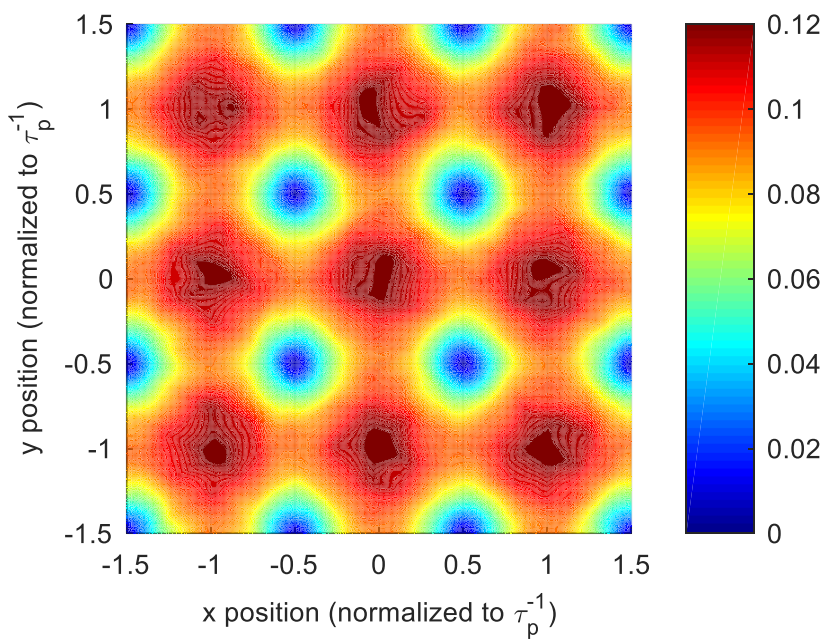

Figure 6: FEA $|\mathbf{B}|$ distribution at $\mathrm{z}=\mathrm{z}_{\mathrm{m}}+\left(\mathrm{z}_{\mathrm{g}}+\mathrm{z}_{\mathrm{c}}\right) / 2$ of internal $3 \times 3$ magnets. 
1

2

3

4

5

6

7

8

9

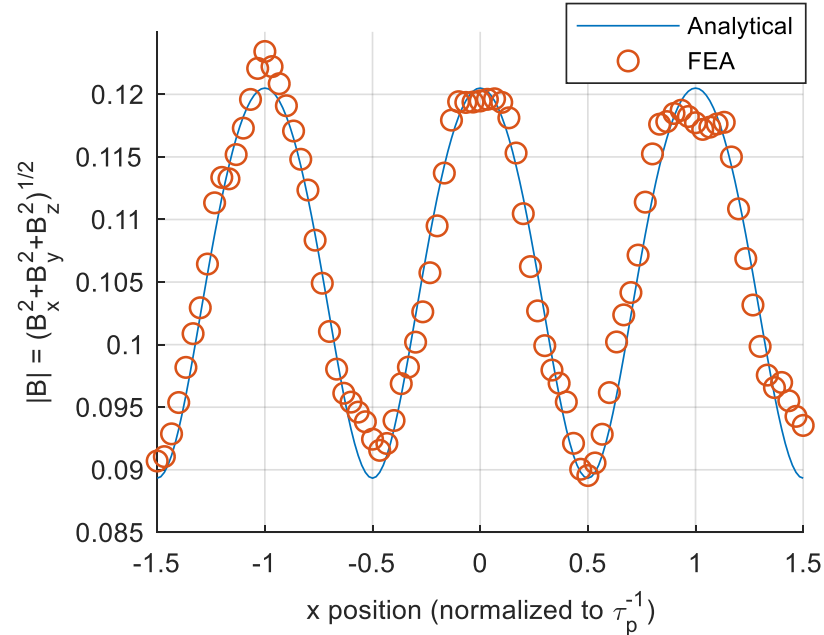

Figure 7: Analytical $|\mathbf{B}|$ distribution at $\mathrm{z}=\mathrm{z}_{\mathrm{m}}+\left(\mathrm{z}_{\mathrm{g}}+\mathrm{z}_{\mathrm{c}}\right) / 2$ of internal $3 \times 3$ magnets. Cross-section at $\mathrm{y}=0$.

$\Phi_{c y z}$ can be found in a similar way as $\Phi_{c x z}$. Hence, the total flux linkage can be found as:

$$
\Phi_{T O T}=\Phi_{c x y}+\Phi_{c x z}+\Phi_{c y z}
$$

To find the induced EMF we first apply a Galilean transform to determine the relative position in terms of the initial coordinates $\left(x_{0}, y_{0}\right)$ and the linear velocities $v_{x}$ and $v_{y}$, i.e. as $x=v_{x} t+x_{0}$ and $y=v_{y} t+y_{0}$. The resulting transformed system can hence be used in conjunction with Faraday's law to find the induced EMF:

$$
E M F=-\frac{d \Phi_{T O T}}{d t}
$$

\section{FEM SOLUTION AND COIL OPTIMIZATION}

\section{A. Magnetic Field Distribution Comparison}

The analytical predictions have been verified using a threedimensional finite-element model. The geometric ratios and magnetic parameters (typical of $\mathrm{NdFeB} 35$ permanent magnets) used in the analysis are per Table. 2.

A 7x7 magnet array was utilized in the simulation to obtain the static field distribution. Natural boundaries were imposed at the interface between objects and Neumann boundaries at the boundaries of the solution domain. The use of natural boundary conditions as opposed to periodic conditions means that non-ideal magnetic end and fringing effects will be present in the simulated solution. Arguably, by verifying the analytical prediction (which neglects such conditions) via these simulated outputs, a complete understanding as to how robust the analytical equations are will be obtained. The primary effect experienced due to these conditions is a net increase in the field magnitude near the edge of the translator array. Considering this, the analysis evaluating the accuracy of the analytical model is based on the data collected from the response due to the magnets internal to the array. This minimizes the effects due to field distortion at the edge of the array and provides insight as to the dominant characteristics (providing the internal magnets are more numerous than the number of magnets at the edge of the array).

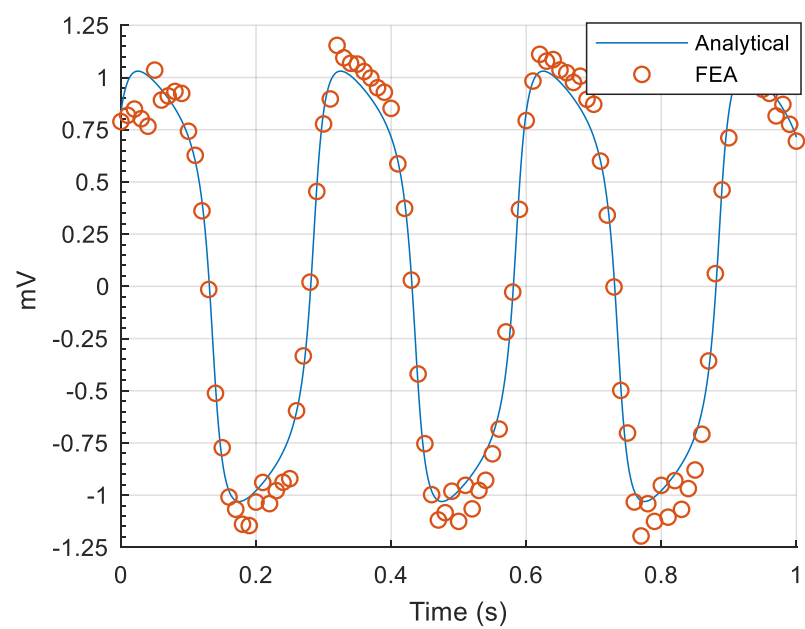

Figure 8: Analytical versus FEA EMF for a single lower coil winding. Geometry ratios: $\tau_{w p x} / \tau_{m}=0.88, \tau_{c p} / \tau_{w p x}=0.132, \tau_{w p x} / \tau_{w p y}=1$, $\tau_{c p} / \tau_{w z}=1, Z_{g} / \tau_{m}=0.067 . v_{x}=0.1 \mathrm{~m} / \mathrm{s} . v_{y}=0 \mathrm{~m} / \mathrm{s}$.

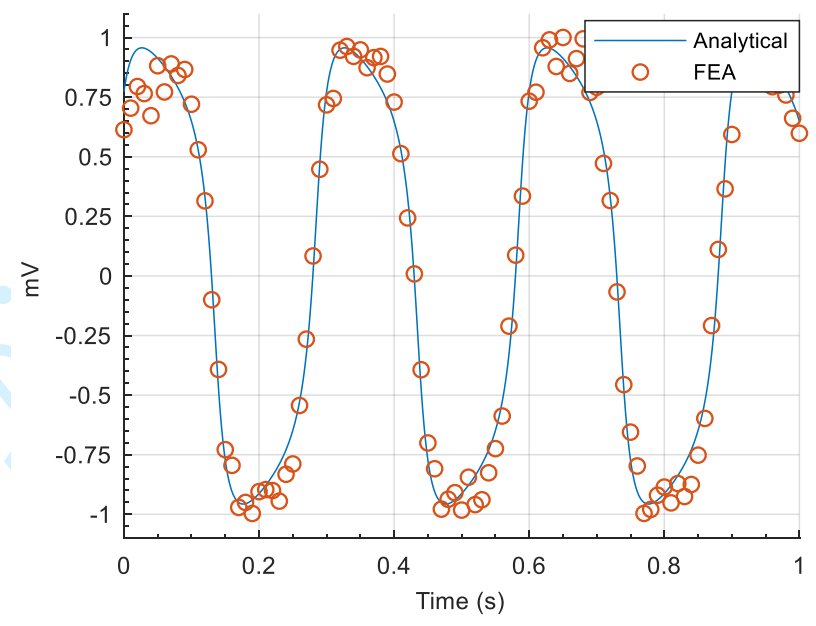

Figure 9: Analytical versus FEA EMF for a single lower coil winding. Geometry ratios: $\tau_{w p x} / \tau_{m}=0.92, \tau_{c p} / \tau_{w p x}=0.091, \tau_{w p x} / \tau_{w p y}=1$, $\tau_{c p} / \tau_{w z}=1, Z_{g} / \tau_{m}=0.067 . v_{x}=0.1 \mathrm{~m} / \mathrm{s} . v_{y}=0 \mathrm{~m} / \mathrm{s}$.

Fig. 5 and 6 respectively show the analytically derived and finite-element simulation magnetic field magnitude at a $Z$ height of $\mathrm{z}=\mathrm{z}_{\mathrm{m}}+\left(\mathrm{z}_{\mathrm{g}}+\mathrm{z}_{\mathrm{c}}\right) / 2$ (i.e. half way through region I), while Fig. 7 illustrates a cross-sectional comparison at $y=$ 0 . The analytical outputs were evaluated up to the $25^{\text {th }}$ harmonic at which point the remaining harmonic magnitudes become negligible.

From each of the figures, it is apparent that there is a reliable correlation, even in the presence of propagating field distortion over the translator due to the end effects. Due to the strong link for the dominant internal magnets, it can be safely asserted that the ideal analytical expressions provide an accurate foundation for rapid initial design evaluation before the use of more detailed finite-element-analysis (FEA). However, caution should be exercised when small order arrays are used (e.g. less than $5 \times 5$ - exclusive) as the end effects will have significant distortion "bleeding" into the internal array magnets. 


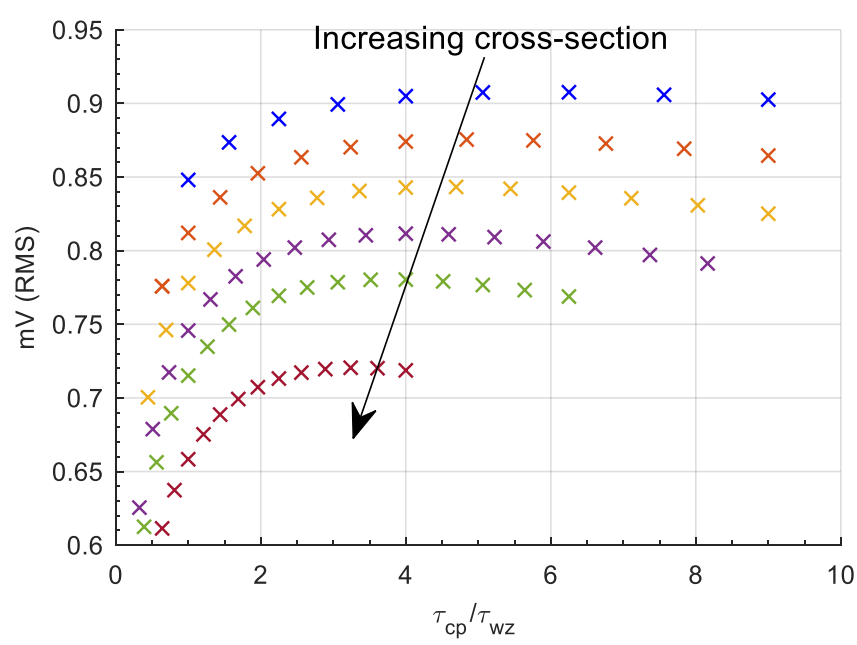

Figure 8: EMF output variation for different $\tau_{c p} / \tau_{w z}$ ratios for a given coil cross-sectional area.

\section{B. EMF Comparison}

Simulations were then conducted using the same finiteelement model from the previous section to verify the presented EMF analytical model. Two simulation outputs are presented in Fig. 8 and 9 for different coil geometries to ensure robustness. In both figures a single coil winding has been utilized as a normalized baseline along with motion in only a single dimension, this has been done to enhance the clarity of the presentation without affecting the overarching average flux theory over the coil space.

The figures presented show a robust correlation between the analytical model and the finite-element simulations, with the peak error for each output being below 10\%. This is particularly in the light that the model has used natural boundaries and hence, a certain degree of uncertainty is present due to the end effects propagating through the field distribution. Nonetheless, even in the presence of this effect, the "ideal" modelling holds to an acceptable accuracy.

An interesting effect that is evident in the presented outputs is that the flux contribution due to $\Phi_{c x z}$ is significant, creating the sharp peak on the leading edge of the waveform, hence illustrating the value in analyzing the output including the side surfaces as well as the primary surface of the coil.

\section{Coil EMF Optimization}

For design, it is desirable to maximize the EMF production per coil winding to avoid extraneous inductance which will compromise the machines efficiency/power-factor. A typical guideline to follow is to ensure that the coil captures the maximum amount of flux per unit area, to do this the coils should be placed as close as possible to the magnets, as the field distribution effectively decreases at an exponential rate from the magnets, and the coil dimensions should be matched strictly to odd integer multiples of $\tau_{p}$; this avoids flux cancellation from the periodic change in field direction (e.g. for a rectangular coil the outer $\mathrm{x}$ dimension could be $3 \tau_{p}$ while the y dimension is $\tau_{p}$ ).

Given a finite coil cross-section, it would be prudent to analyze the optimal ratio of vertical to horizontal coil pitch.

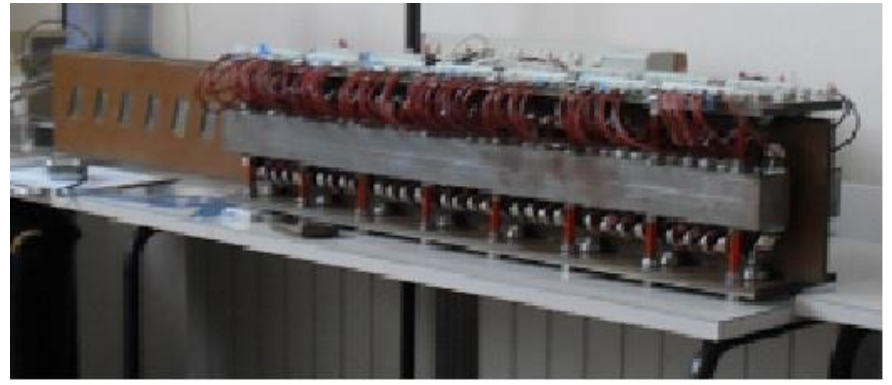

Figure 7: The linear generator built. The translator with a skewing of $17^{\circ}$ is shown.

By way of example, using the established analytical model, consider a coil from the lower layer of the herringbone structure which has a prescribed cross-sectional area $A$. By varying the coil dimensions such that $\tau_{c p} \tau_{w z}=A$ the ideal ratio between the horizontal and vertical dimensions can be established.

Fig. 10 illustrates the output voltage for an arbitrary single coil winding for a range of different cross-sectional areas with respect to the ratio of $\tau_{c p}$ and $\tau_{w z}$. The figure shows that $\tau_{c p}$ generally should range from 2 to 5 times $\tau_{w z}$, being on the upper end for low cross-sections and vice versa. Ideally, this optimization process should be used in an iterative development process to ensure the most effective use of the coil space. This may proceed by finding a convergence between the coil area and the area required by the coils through variation of the back-iron height $z_{s}$ and then reevaluating the optimal coil geometry to suit the available space.

\section{EXPERIMENTAL RESULTS}

In order to evaluate the efficacy of the proposed analysis, the mathematical model has been tested on a one-dimensional prototype generator (see Fig.11). The generator has been already studied under a different mathematical model in.

The mathematical approach that has been followed is based on a one-dimension adaptation of the proposed threedimensional model. Mechanical, geometric, and electrical parameters are per Table.III for the experimental machine.

In the following subsections, we analyse and validate the analytical model against results obtained from the hardware generator.

\section{A. Output voltage}

Fig.12 presents an illustration comparing the theoretically predicted output voltage from the model versus the actual voltage from the experimental hardware. Under experimental conditions the machine was driven by a falling mass connected to the translator through a pulley system achieving an average speed of $1 \mathrm{~m} / \mathrm{s}$; hence we observe an increasing voltage output as the velocity increases. The analytical model utilized measured velocity data to match the experimental result.

Overall, it can be seen that the theoretical estimation of the output satisfactorily predicts the actual output from the experimental hardware. The peak output amplitudes are accurately computed from the analytical model; however, the 


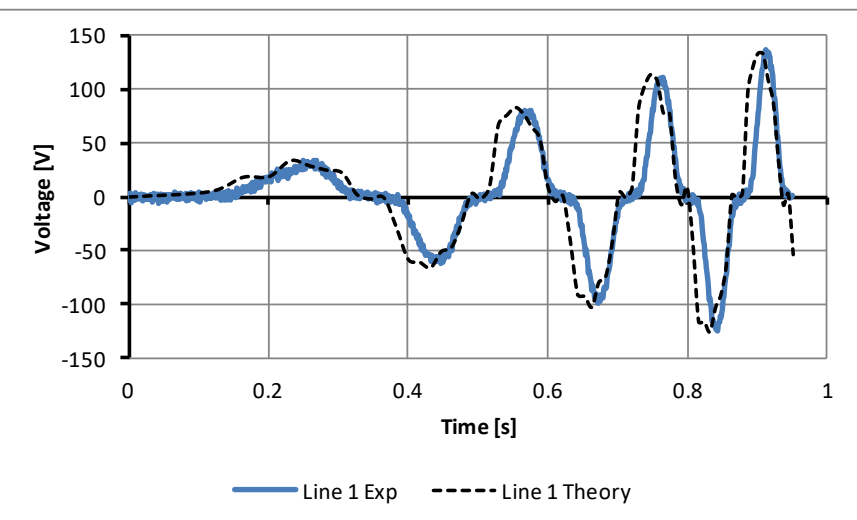

Figure 9: A comparison between a theoretical and measured output voltage for the translator moving under a constant acceleration.

TABLE III LINEAR GENERATOR PARAMETERS

\begin{tabular}{|c|c|}
\hline Machine section & Parameters \\
\hline Stator & $\begin{array}{l}\text { - } \text { Size: } 60 \times 972 \times 65 \mathrm{~mm} \\
\text { - } 126 \text { iron sheets } \\
\text { - } \quad \text { Sheet's thickness } 0.5 \mathrm{~mm} \\
\text { - } 39 \text { slots } \\
\text { - } \quad \text { Internal slot width: } 12 \mathrm{~mm} \\
\text { - } 8 \text { holes (diameter } 10,5 \mathrm{~mm} \text { ) for the tightening } \\
\text { of the sheets with bolts }\end{array}$ \\
\hline Mover & $\begin{array}{l}\text { - Alternating magnets interspersed with soft } \\
\text { iron poke pieces mounted on a Bakelite sheet. } \\
\text { - Sheet size: } 1600 \times 11 \times 206 \mathrm{~mm}\end{array}$ \\
\hline Windings & $\begin{array}{l}\text { - } \text { Enameled copper wire } 0.5 \mathrm{~mm} \text { diameter } \\
\text { - } 375 \text { turns } \\
\text { - } \text { Rectangular shape } \\
\text { - } 85 \times 135 \mathrm{~mm} \\
\text { - } \quad \text { Coil weight: } 278 \mathrm{~g}\end{array}$ \\
\hline $\begin{array}{l}\text { Permanent } \\
\text { magnets }\end{array}$ & $\begin{array}{l}\text { - } \mathrm{Ne}-\mathrm{Fe}-\mathrm{B} \\
\text { - } \quad 40 \text { magnets } \\
\text { - } \quad \text { Size: } 60 \times 30 \times 15 \mathrm{~mm} \\
\text { - } \quad \text { Weight: } 205.2 \mathrm{~g}\end{array}$ \\
\hline
\end{tabular}

model lacks the detail to describe additional cogging forces which are exhibited in the experimental trace of Fig.12. This is as expected due to the slottless and periodic geometry used for the basis of analysis.

It is worth noting that the mathematical model was scaled to match the experimental hardware which utilized 6 coils in series, each with 1000 turns, to achieve acceptable output magnitudes.

\section{B. Magnetic field}

In Fig.13 we compare the behavior of the magnetic field measured in the air gap of the generator with the theoretical one computed by using the proposed mathematical model. The analytical model suitably describes the general behaviour of the magnetic field. Similarly to before, irregularities in the field from various non-periodic fringing effects and slotting in the structure which can lead to intense cogging forces are not depicted in the analytical model.

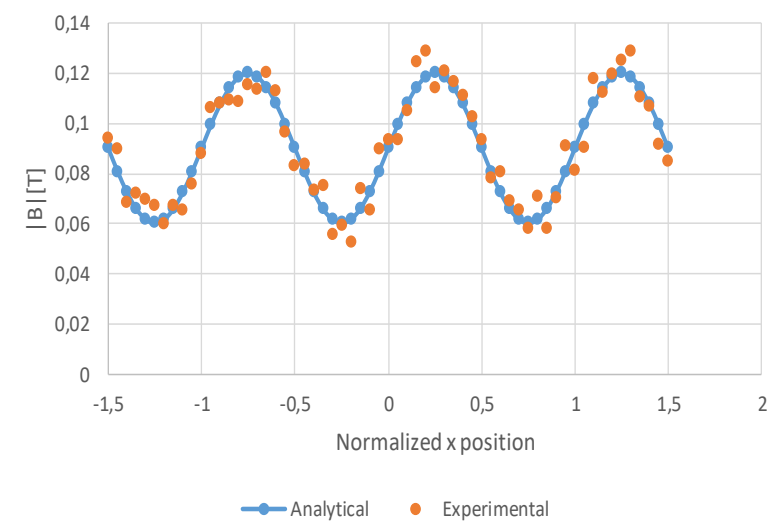

Fig.13 Comparison between analytical and experimental values of magnetic field of magnets. Cross-section at $\mathrm{y}=0$.

\section{CONCLUSION}

In this paper, a planar generator for a wave energy conversion system has been described. The proposed generator is capable of producing electrical power along two axes and therefore in an inertial generator installed in a floating body can exploit both rolling and pitching motion. The principle of operation and geometric requirements of the generator has been presented along with an analytical model. This model has been validated against a FEM numerical analysis and several experimental results obtained by using a one dimensional linear generator.

\section{ACKNOWLEDGMENT}

This work was partly funded by the Australian Government under the project ARC DP170101039.

This work was partly funded from the activities n. 7,11 and 12 jointly developed in the year 2013 with SGES S.r.l. by one of the author.

\section{REFERENCES}

[1] V. Boscaino, G. Cipriani, V. Di Dio, V. Franzitta, and M. Trapanense, "Experimental Test and Simulations on a Linear Generator-Based Prototype of a Wave Energy Conversion System Designed with a Reliability-Oriented Approach," Sustainability, vol. 9 (1), 2017.

[2] A. F. de O. Falcão, "Wave energy utilization: A review of the technologies," Renewable and Sustainable Energy Reviews, vol. 14, no. 3, pp. 899-918, 2010.

[3] A. Clément, P. McCullen, A. Falcão, A. Fiorentino, F. Gardner, K. Hammarlund, G. Lemonis, T. Lewis, K. Nielsen, S. Petroncini, M.-T. Pontes, P. Schild, B.-O. Sjöström, H. C. Sørensen, and T. Thorpe, "Wave energy in Europe: current status and perspectives," Renewable and Sustainable Energy Reviews, vol. 6, no. 5, pp. 405-431, 2002.

[4] J. Brooke, Wave energy conversion, vol. 6. Elsevier, 2003.

[5] J. F. Pan, S. Y. Li, E. Cheng, and B. Zhang, "Analysis of a Direct Drive 2-D Planar Generator for Wave Energy Conversion," IEEE Trans. Magn., vol. 53, no. 11, pp. 2-6, 2017.

[6] R. Crozier and M. Mueller, "Integrated structural and electromagnetic design of direct-drive linear machines for wave energy," IET Renew. Power Gener., vol. 6, no. 3, pp. 137-148, 2012

[7] J. W. Jansen, J. P. C. Smeets, T. T. Overboom, J. M. M. Rovers, and E. A. Lomonova, "Overview of Analytical Models for the Design of Linear and Planar Motors", IEEE Trans. On magnetics, Vol. 50, NO. 11, Nov 2014. 
[8] J. Cao, Y. Zhu, and J. Wang, "A novel synchronous permanent magnet planar motor and its model for control applications," IEEE Trans. Magn., vol. 41, no. 6, pp. 2156-2163, Jun. 2005.

[9] M. Trapanese, V. Boscaino, G. Cipriani, D. Curto, V. Di Dio, and V. Franzitta, "A Permanent Magnet Linear Generator for the Enhancement of the Reliability of a Wave Energy Conversion System," IEEE Trans. Ind. Electron., vol. 0046, no. c, pp. 1-1, 2018.

[10] J. W. Jansen, C. M. M. Van Lierop, E. A. Lomonova, S. Member, A. J. A. Vandenput, and S. Member, "Magnetically Levitated Planar Actuator With Moving Magnets," IEEE Trans. Ind. Appl., vol. 44, no. 4, pp. 1108-1115, 2008.

[11] H.-S. Cho, C.-H. Im, and H.-K. Jung, "Magnetic field analysis of 2-D permanent magnet array for planar motor," IEEE Trans. Magn., vol. 37, pp. 3762-3766, Sept. 2001. 